\title{
The potential of cerium oxide nanoparticles (nanoceria) for neurodegenerative disease therapy
}

\author{
"The use of nanoceria for therapeutic purposes provides multiple advantages over \\ other novel antioxidant approaches."
}

Keywords: cerium oxide $\bullet$ nanoceria $\bullet$ neurodegenerative disease $\bullet$ oxidative stress

Free radicals, molecules with an unpaired electron in the outer orbital shell, are highly reactive and strip electrons from biomolecules such as proteins, lipids and DNA, causing cellular damage. Since they can be formed as normal byproducts of cellular respiration, cells have endogenous antioxidant systems, such as enzymes like catalase and superoxide dismutase (SOD), to help minimize damage due to rogue free radicals. However, under pathological conditions, the production of free radicals can exceed the capacity of endogenous systems to neutralize them, resulting in a condition known as oxidative stress. Oxidative stress has been shown to play a role in aging and in the pathology of stroke and many neurodegenerative diseases [1]. Although we have a good understanding of the role of free radicals and oxidative stress in these disorders, there is still a paucity of antioxidants that have proven effective for treating neurodegenerative diseases.

$\mathrm{CeO}_{2}$ nanoparticles (nanoceria) have the potential to be developed as a therapeutic for oxidative stress diseases due to their catalytic antioxidant properties. Cerium is a rare earth metal that, when combined with oxygen, can adopt a fluorite crystalline lattice structure that has a highly reactive surface area for neutralization of radicals. In addition, nanoceria can reversibly bind oxygen and shift oxidation states $\left(\mathrm{Ce}^{3+} / \mathrm{Ce}^{4+}\right)$ depending on the conditions. Because of these properties, nanoceria have been studied in biological systems and shown to display antioxidant effects in various models of disease [2]. For example, we have shown the neuroprotective effects of nanoceria using a hippocampal brain slice model of cerebral ischemia [3], as well as a murine model of multiple sclerosis, experimental autoimmune encephalomyelitis [4].

The use of nanoceria for therapeutic purposes provides multiple advantages over other novel antioxidant approaches. For example, the delivery of nanoencapsulated antioxidant enzymes, such as SOD or catalase, has the limitation that only one type of reactive oxygen species can be scavenged by each enzyme, whereas, multiple species are implicated in neurodegenerative diseases [1] and nanoceria have been shown to decrease their levels [3,5]. In addition, there is a limited amount of enzyme that can be delivered within the nanostructure. In contrast, nanoceria exhibits both catalaseand SOD-mimetic activity with SOD-mimetic catalysis exceeding that of the endogenous enzyme $[6,7]$. One study showed that $5.8 \mu \mathrm{M}$ nanoceria was equivalent to $527 \mathrm{U}$ of $\mathrm{SOD}[8]$. Alternatively, activation of endogenous antioxidants via the Nrf-2 pathway has also been pursued. Nrf-2 is a transcription factor that mediates the activation of a suite of antioxidant genes, including genes that encode catalase, SOD and several proteins involved in glutathione homeostasis, via the antioxidant response element [9]. Indeed, synthetic triterpanoids that function as activators of the Nrf-2/antioxidant response element pathway have been shown effective in models of neurodegenerative disease [10]. However, this strategy may be limited under pathological conditions in which increased gene expression alone is not sufficient to mitigate the increase in oxidative stress.

Despite these advantages of nanoceria over other therapeutic strategies, there are toxicity issues to consider. In particular, pro-oxidant

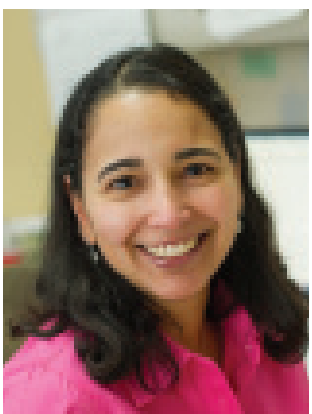

Ana Y Estevez

Author for correspondence: Department of Biology, St. Lawrence University, Canton, NY 13617, USA and Department of Psychology, St. Lawrence University, Canton, NY 13617, USA aestevez@stlawu.edu

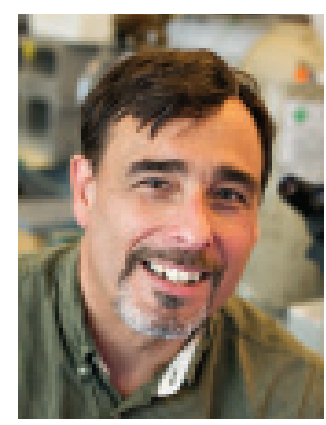

Joseph S Erlichman Department of Biology, St. Lawrence University, Canton, NY 13617, USA

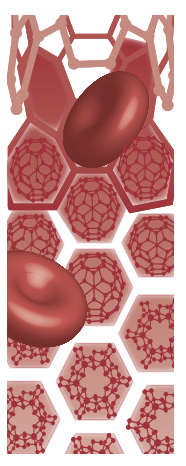


effects of cerium oxide nanoparticles have been reported in various model systems [11,12], and this would be cause for concern if the ultimate goal is to translate their use to a clinical setting. However, cerium oxide nanoparticles, although comprised of the same core elements, do not all display the same biological effects. We hypothesize that disparate reports - of pro-oxidant toxicity in some cases and antioxidant protective effects in others - could be explained by the different physiochemical parameters of the various nanoparticles tested, such as method of synthesis, particle size and stabilizer. These parameters can then influence the biological identity of the particle due to the adsorption of various proteins on the nanoparticle surface. Differing biological identities could lead to varying interactions with cellular constituents, affecting the biodistribution of the particles and their ultimate effects on the organism. We will explore these ideas further in the sections below.

\section{Role of synthesis, particle size \& stabilizer}

Different synthesis methods can endow cerium oxide nanoparticles with varying physiochemical and catalytic properties that could contribute to pro-oxidant or antioxidant properties, as Dowding et al. recently demonstrated [13]. Another complication is that the properties of the particle measured in vitro (e.g., size, zeta potential and redox activity) could change under physiological conditions. For example, Kumari et al. showed that the hydrodynamic diameter nanoceria increased dramatically in cell culture media due to the tendency of the particles to agglomerate in physiological buffers [12]. In addition, adsorption of proteins present in biological fluids, such as blood, can also influence the size and distribution of metal oxide nanoparticles [14,15].

\section{"Many particles have been synthesized with a coating or stabilizer with the ultimate goal of yielding monodispersed particles that are less."}

Many particles have been synthesized with a coating or stabilizer with the ultimate goal of yielding monodispersed particles that are less prone to aggregation while still retaining the chemistry inherent in the particle. Citric acid is a commonly used stabilizer due to its biocompatibility. However, it is important to note that the stabilizer too can change once immersed into physiological solution. Specifically, citrate-stabilized particles have been shown to precipitate or salt-out in high ionic strength solutions [16].

\section{Biological identity of nanoparticles}

Studies looking at the physiochemical characteristics of nanoparticles after interaction with biological fluids provide some insight into the role of protein-nanoparticle interactions in ultimate nanoparticle effects. Walczyk et al. incubated various types of nanoparticles with human blood plasma and showed that plasma proteins can interact with the nanoparticles to form a stable, long-lasting corona on the particle surface [17]. Walkey et al. systematically analyzed the protein corona of 105 different surface-modified gold nanoparticles [18]. Incubation of these nanoparticles with undiluted human serum led to a different set of proteins adsorbed onto the nanoparticle surface, a so-called corona fingerprint for each type of particle, which then either promoted or inhibited the interaction of the particle with human lung carcinoma cells. Taken together, these and other studies suggest that the protein corona endows the nanoparticle with a particular biological identity that subsequently plays an important role in the ultimate interactions of a nanoparticle with target cells. A protein corona could, for example, induce novel surface receptor interactions, influence the intracellular targeting of the particle to specific organelles or trigger immune cell activation $[15,19]$. Although a systematic analysis of the protein corona fingerprint of cerium oxide nanoparticles has yet to be conducted, one can extrapolate these general conclusions to help interpret the varying effects of nanoceria in whole animals observed in some recent studies $[4,11,20]$.

\section{Sorting out the effects of nanoceria in whole animals}

Using the experimental autoimmune encephalomyelitis model of multiple sclerosis, we recently showed that custom-synthesized citrate/ethylenediaminetetraacetic acid (EDTA)-stabilized nanoceria reduced free radical levels in the brain and alleviated the clinical symptoms and motor deficits associated with disease progression. The mice received multiple doses of the particles (up to $30 \mathrm{mg} / \mathrm{kg}$ ) over a 35 -day period. We used the same particles in a G93A mouse model of amyotrophic lateral sclerosis and observed dose-dependent improvement in motor function and survival when particles were delivered at the onset of motor symptoms (Erlichman et al., unpublished observations). However, in a long-term study, Hardas et al. measured significant increases in multiple oxidative stress parameters in rat hippocampus after single intravenous infusion $(87 \mathrm{mg} / \mathrm{kg})$ of a $30 \mathrm{~nm}$ citrate-stabilized nanoceria, even though the nanoceria did not penetrate the brain [11]. Oxidative stress was elevated for up to 30 days post-infusion, but most parameters returned back to baseline by 90 days.

One possibility to account for these differing reports is the concentration of the nanoparticles used, as toxicological studies typically use higher doses. Another possibility is that in the studies where nanoceria displayed toxicity, the particles agglomerated in the blood if the stabilizer precipitated out [16] or bound different protein 
coronas, both of which could lead to an enhanced immune response. Indeed, hippocampal oxidative stress was observed with minimal detection of nanoceria in the brain but high accumulation in reticuloendothelial organs, such as the liver and spleen, as well as increases in the levels of interleukin-1 beta, an inflammatory cytokine $[11,20]$. On the other hand, the particles used in the study by Heckman et al. were stabilized with a customsynthesized mixture of citrate/EDTA and TEM analysis demonstrated that these particles had an average size of $2.9 \mathrm{~nm}$, were monodispersed even in high salt solutions, and could be detected in the brain following intravenous administration [4].

\section{Conclusion}

The core redox chemistry of nanoceria presents multiple advantageous properties for the treatment of oxidative stress diseases. However, an important consideration in their clinical translation is how cerium oxide nanoparticles behave in biological systems. Addressing this is not a simple endeavor. Rational design of surface coatings

\section{References}

1 Estevez AY, Erlichman JS. Cerium oxide nanoparticles for the treatment of neurological oxidative stress diseases. In: Oxidative Stress: Diagnostics, Prevention and Therapy (Volume 1083). Andreescu ES, Hempel $\mathrm{M}(\mathrm{Eds})$. American Chemical Society, Washington, DC, USA, 255-288 (2011).

2 Das S, Dowding JM, Klump KE, McGinnis JF, Self W, Seal S. Cerium oxide nanoparticles: applications and prospects in nanomedicine. Nanomed. (Lond.) 8(9), 1483-1508 (2013).

3 Estevez AY, Pritchard S, Harper K et al. Neuroprotective mechanisms of cerium oxide nanoparticles in a mouse hippocampal brain slice model of ischemia. Free Radic. Biol. Med. 51(6), 1155-1163 (2011).

4 Heckman KL, DeCoteau W, Estevez A et al. Custom cerium oxide nanoparticles protect against a free radical mediated autoimmune degenerative disease in the Brain. ACS Nano 7(12), 10582-10596 (2013).

5 Hirst SM, Karakoti AS, Tyler RD, Sriranganathan N, Seal S, Reilly CM. Anti-inflammatory properties of cerium oxide nanoparticles. Small 5(24), 2848-2856 (2009).

6 Pirmohamed T, Dowding JM, Singh $S$ et al. Nanoceria exhibit redox state-dependent catalase mimetic activity. Chem. Commun. (Camb.) 46(16), 2736-2738 (2010).

7 Korsvik C, Patil S, Seal S, Self WT. Superoxide dismutase mimetic properties exhibited by vacancy engineered ceria nanoparticles. Chem. Commun. (Camb.) 10(10), 1056-1058 (2007).

8 Ganesana M, Erlichman JS, Andreescu S. Real-time monitoring of superoxide accumulation and antioxidant activity in a brain slice model using an electrochemical cytochrome c biosensor. Free Radic. Biol. Med. 53(12), 2240-2249 (2012). may mitigate some of the agglomeration issues in vitro, but may not be predictive of the whole animal condition. As the nanoparticles traverse multiple biological compartments, each with varying ionic and protein compositions, some coatings may become destabilized. In addition, different repertoires of proteins may adsorb onto the nanoparticle surface, ultimately driving the biological effects. Since there are currently no unifying principles for predicting these effects, each particle must therefore be individually characterized in a biological setting.

Financial \& competing interests disclosure

AY Estevez and JS Erlichman both have less than 15\% equity ownership in Cerion NRx, which funded a portion of this work. The authors have no other relevant affiliations or financial involvement with any organization or entity with a financial interest in or financial conflict with the subject matter or materials discussed in the manuscript apart from those disclosed.

No writing assistance was utilized in the production of this manuscript.

9 Kang KW, Lee SJ, Kim SG. Molecular mechanism of nrf2 activation by oxidative stress. Antioxid. Redox Signal. 7(11-12), 1664-1673 (2005).

10 Neymotin A, Calingasan NY, Wille E et al. Neuroprotective effect of Nrf2/ARE activators, CDDO ethylamide and CDDO trifluoroethylamide, in a mouse model of amyotrophic lateral sclerosis. Free Radic. Biol. Med. 51(1), 88-96 (2011).

11 Hardas SS, Sultana R, Warrier G et al. Rat hippocampal responses up to 90 days after a single nanoceria dose extends a hierarchical oxidative stress model for nanoparticle toxicity. Nanotoxicology 1-12 (2013).

12 Kumari M, Singh SP, Chinde S, Rahman MF, Mahboob $\mathrm{M}$, Grover P. Toxicity study of cerium oxide nanoparticles in human neuroblastoma cells. Int. J. Toxicol. 33(2), 86-97 (2014).

13 Dowding JM, Das S, Kumar A et al. Cellular interaction and toxicity depend on physicochemical properties and surface modification of redox-active nanomaterials. ACS Nano 7(6), 4855-4868 (2013).

14 Deng ZJ, Liang M, Monteiro M, Toth I, Minchin RF. Nanoparticle-induced unfolding of fibrinogen promotes Mac-1 receptor activation and inflammation. Nat. Nanotechnol. 6(1), 39-44 (2011).

15 Deng ZJ, Mortimer G, Schiller T, Musumeci A, Martin D, Minchin RF. Differential plasma protein binding to metal oxide nanoparticles. Nanotechnology 20(45), 455101 (2009).

16 Levy R, Thanh NTK, Doty RC et al. Rational and combinatorial design of peptide capping ligands for gold nanoparticles. J. Am. Chem. Soc. 126(32), 10076-10084 (2004).

17 Walczyk D, Bombelli FB, Monopoli MP, Lynch I, Dawson KA. What the cell "sees" in bionanoscience. J. Am. Chem. Soc. 132(16), 5761-5768 (2010). 
18 Walkey CD, Olsen JB, Song F et al. Protein corona fingerprinting predicts the cellular interaction of gold and silver nanoparticles. ACS Nano 8(3), 2439-2455 (2014).

19 Asati A, Santra S, Kaittanis C, Perez JM. Surface-chargedependent cell localization and cytotoxicity of cerium oxide nanoparticles. ACS Nano 4(9), 5321-5331 (2010).
20 Hardas SS, Sultana R, Warrier G et al. Rat brain prooxidant effects of peripherally administered $5 \mathrm{~nm}$ ceria 30 days after exposure. Neurotoxicology 33(5), 1147-1155 (2012). 